\title{
Le cyber-diable se cache aussi dans les détails...
}

\section{Urs Stoffel}

Dr, membre du Comité central de la FMH, responsable du département eHealth - Infrastructure de sécurité et collecte des données

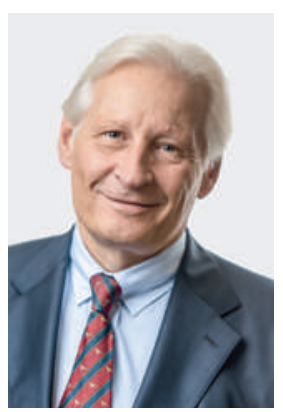

... et ils sont nombreux dans le droit d'exécution de la loi fédérale sur le dossier électronique du patient, en audition depuis fin mars 2016. Le groupe de travail «eHealth» de la FMH a pris position en première lecture sur l'ensemble d'ordonnances relatives au dossier électronique du patient.

Ce qui frappe à première vue, c'est le nombre et l'étendue des détails du projet. Pour le groupe de travail, ces ordonnances remplissent toutes les conditions pour «asphyxier» le dossier électronique du patient.

Si les différents standards font l'objet de descriptions détaillées, aucune précision n'est donnée concernant les processus permettant de les mettre en place. Or pour que le dossier électronique du patient soit viable, ce ne sont pas les détails techniques qui importent mais bien les processus.

Par ailleurs, les obstacles d'ordre technique et organisationnel sont en partie très élevés et compliquent l'introduction du dossier électronique. Les expériences faites à Genève dans le cadre du projet «MonDossierMedical» montrent cependant qu'un accès facilité pour toutes les parties - sans pour autant compromettre la sécurité - est primordial pour la diffusion du dossier. De plus, plusieurs dispositions vont à l'encontre des intérêts des patients, car elles ne permettent pas de

\section{Les données de patients utiles pour de futurs traitements doivent être conservées.}

garantir la sécurité et la continuité du traitement. Les dispositions relatives à l'organisation notamment semblent en partie éloignées de la réalité et donc difficilement applicables. Un exemple: au lieu d'insister sur la destruction des données des dossiers électroniques dans le délai prévu, il faudrait plutôt mettre davantage la priorité sur la conservation des données utiles pour de futurs traitements. Pour la FMH, il ne fait aucun sens de détruire les données d'un cabinet qui cesse son activité dans les différents dossiers électroniques ou qui, pour toute autre raison, ne renouvelle pas sa participation à une communauté. Comme pour les dispositions prévues lors de la fermeture d'un cabinet, il serait bien plus judicieux d'édicter des dispositions nationales; ne serait-il pas plutôt nécessaire de mettre sur pied une institution supplétive se chargeant des données de patients, d'une part pour les membres des communautés et de l'autre, pour les communautés qui ne seraient plus reconduites?

\section{Le nombre et l'étendue des dispositions} risquent d'asphyxier le dossier électronique du patient.

Selon le groupe de travail de la FMH, le projet dissuade ceux qui envisagent de créer une communauté. Probablement, seuls quelques cantons suffisamment riches pourront se permettre une telle dépense, du moins pour la constitution d'une communauté de référence. Tous ces points qui suscitent la critique font craindre l'émergence de «mondes parallèles», ce qui ne serait pas dans l'intérêt des patients.

La FMH participe très volontiers, dans la mesure de ses possibilités, à l'élaboration d'un dossier électronique du patient applicable au quotidien, qui soit viable et puisse s'adapter à l'évolution constante de la médecine mais aussi des besoins en matière de documentation et de communication. Elle s'engage pour un dossier électronique qui place le patient au centre et qui le soutient dans son traitement. Pour la FMH, le dossier électronique du patient doit être conçu de manière à instaurer un espace sûr - car de la confiance dépendent le succès et la sécurité du traitement. 